in the development of pneumonia associated with viruses and that these viruses contribute to the pathogenesis of bacterial pneumonia.

In conclusion, this study establishes that a significant proportion of viral pneumonia is attributable to bacterial co-infection and is preventable by a bacterial vaccine. Because immunization of children has been shown to reduce invasive $S$. pneumoniae disease, these data also raise the exciting possibility that infant immunization with pneumococcal vaccines might also reduce morbidity and mortality associated with viral pneumonias in children and adults.

David O'Connell

(4) References and links ORIGINAL RESEARCH PAPER Madhi, S. A. et al. A role for Streptococcus pneumoniae in virus-associated pneumonia. Nature Med. (July 2004) doi:10.1038/nm1077

FURTHER READING Klugman, K. P. et al.

A trial of a 9-valent pneumococcal conjugate vaccine in children with and those without HIV infection. N. Engl. J. Med. 349, 1341-1348 (2003) WEB SITE

Keith Klugman's laboratory:

http://www.sph.emory.edu/ih/kklugman.html

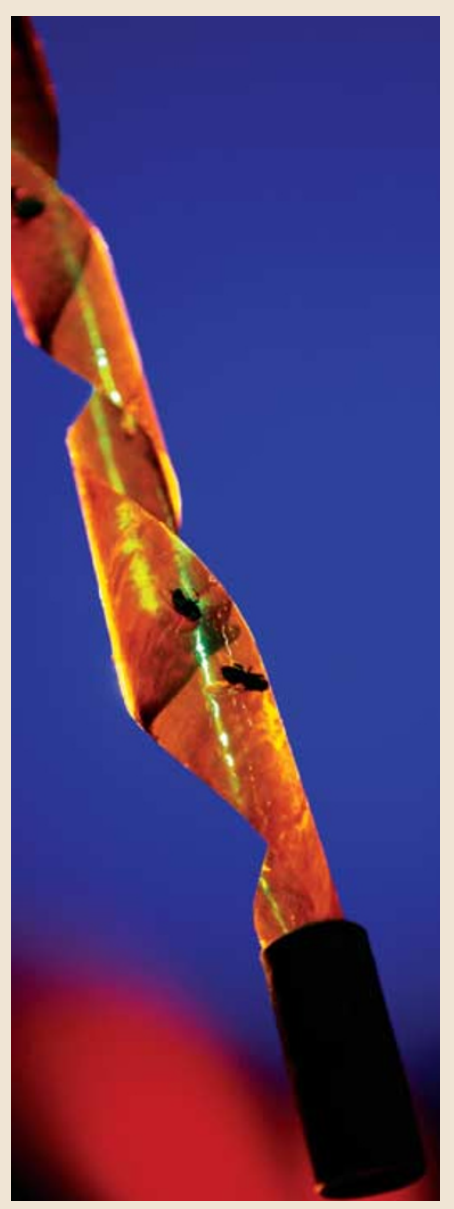

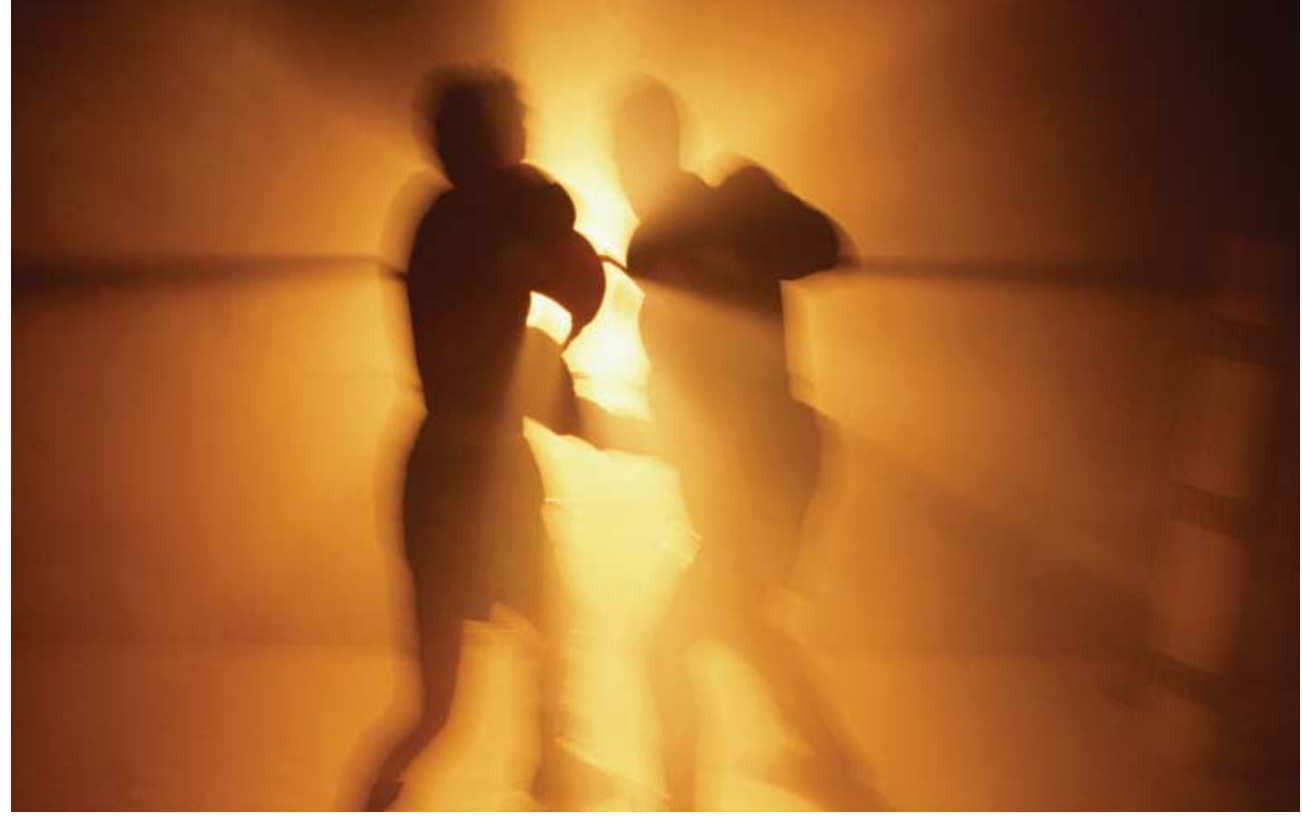

MALARIA

\title{
Joint attack on malaria
}

An international group of scientists, drawn from both academia and industry, has developed a new antimalarial drug candidate that will move into human clinical trials later this year.

The multidisciplinary team, which brought together researchers from the University of Nebraska, USA, Monash University, Australia, The Swiss Tropical Institute, Switzerland, and Hoffman-La Roche, Switzerland, describe in a recent issue of Nature how the early determination of the essential characteristics for a new antimalarial drug guided the discovery process, and ultimately enabled the selection of a new synthetic peroxide drug candidate with an improved therapeutic and pharmaceutical profile compared with artemisinin.

Malaria is caused by unicellular parasites of the Plasmodium genus, which can be transmitted to human blood by the bite of the Anopheles mosquito. Artemisinin contains a crucial peroxide bond that is believed to enable the formation of unstable free radicals of the drug, which poison the Plasmodium parasite by alkylating one or more of its essential proteins. Although artemisinin itself and several semisynthetic derivatives are in use today, concerns exist about their high cost of production, low oral activity and the length of treatment required.

The group's goal was to identify a new synthetic peroxide antimalarial with improved oral potency, shorter treatment requirements, low toxicity, and which is cheaper and easier to produce. With these aims in mind, the team developed a clearly defined product profile that guided the lead-optimization process.

First, they established a number of required molecular properties of the compound to ensure adequate oral absorption, such as lipophilicity, polar surface area and solubility. They then selected suitable intermediate compounds, to which functional groups could be added using simple chemical transformations to create candidate compounds having these desired properties. The antimalarial activity of a single dose of each candidate was assessed in vivo using mice infected with Plasmodium berghei, and the rate at which active compounds were broken down was assessed in vitro using microsome preparations containing metabolizing enzymes.

Candidates with good oral bioavailability, high activity and an acceptable half-life were then subjected to more rigorous secondary screens, in which they were compared with currently used malaria treatments, including artemisinin derivatives. Antimalarial activity was assessed in vitro against Plasmodium falciparum, the main cause of severe clinical malaria and death in humans. The $P$. berghei mouse model was also used to assess how quickly the compounds reduced disease symptoms, whether symptoms recurred and whether the compounds could protect against disease when administered before infection. Candidates were also assessed for their susceptibility to metabolism by cytochrome $\mathrm{P} 450$ enzymes, in addition to being subjected to standard toxicity tests.

A candidate has been selected on the basis of these tests that will undergo clinical testing in humans later this year. If successful, it will not only improve treatment for the millions of people in developing countries who contract malaria each year, but will also highlight the value of establishing strict selection criteria early on in the drug discovery process to guide lead optimization. Success would also demonstrate the potential for fruitful collaborations between academia and industry.

Clare Ellis, Associate Editor, Nature Reviews Drug Discovery

\section{(2) References and links}

ORIGINAL RESEARCH PAPER Vennerstrom, J. L. et al. Novel

antimalarial peroxides: identification of a trioxolane drug development candidate. Nature (14 July 2004) doi:10.1038/nature02779 